\title{
AVALIAÇÃO DO MÉTODO DQST NA SOLUÇÃO DA EQUAÇÃO DE BALANÇO POPULACIONAL
}

\author{
G. G. S. FERREIRA ${ }^{1}$, P. L. C. LAGE*1, L. F. L. R. SILVA ${ }^{2}$ \\ ${ }^{1}$ Universidade Federal do Rio de Janeiro, Programa de Engenharia Química - COPPE, \\ Caixa postal 68502, Rio de Janeiro, RJ, 21941-272, Brasil \\ ${ }^{2}$ Universidade Federal do Rio de Janeiro, Escola de Química \\ *tel:+55 212562 8346; fax: +55 212562 8300; e-mail: paulo@peq.coppe.ufrj.br
}

\begin{abstract}
RESUMO
Este trabalho trata da implementação e avaliação do método DQST (Direct Quadrature Spanning Tree Method), para a solução numérica da equação de balanço populacional. Embora este método já tenha sido utilizado na solução de problemas acoplando balanço populacional com dinâmica dos fluidos computacional, falta uma análise mais aprofundada da acurácia do mesmo. Com isso, este trabalho trata do levantamento das atuais limitações do método, através da comparação dos resultados obtidos com soluções analíticas reportadas na literatura. Estes resultados serão utilizados no desenvolvimento de trabalhos futuros, onde modificações ao método DQST serão propostas.
\end{abstract}

\section{INTRODUÇÃO}

A modelagem por balanço populacional (BP) é uma ferramenta versátil na análise de sistemas particulados, já tendo sido aplicada à análise de colunas de borbulhamento, leitos fluidizados, extração líquido-líquido, secagem de sólidos etc. (MARCHISIO e FOX, 2013).

Com isso, diversos métodos numéricos já foram desenvolvidos para a solução da equação de balanço populacional (EBP), sendo os mesmos geralmente classificados em métodos de Monte Carlo, métodos seccionais, métodos baseados em momentos e métodos híbridos, como os métodos de momentos fechados por quadratura, entre outros (RAMKRISHNA, 2000).

Recentemente, Vikhansky (2013) desenvolveu o método DQST, que é um método numérico adaptativo para a solução da equação de balanço populacional (EBP) monovariada e multivariada. Este método possui características tanto dos métodos de discretização, como o método das classes com pivôs móveis de Kumar e Ramkrishna (1996), quanto dos métodos baseados em quadratura, como o DQMoM (MARCHISIO e FOX, 2005). Entretanto, o método DQST tem a vantagem de não necessitar da inversão de uma matriz mal condicionada, que é o caso dos métodos baseados em momentos fechados por quadratura, e nem parte da definição prévia de uma malha para o espaço das variáveis internas da distribuição, que é o caso dos métodos seccionais.

Assim, a principal diferença entre o DQMoM e o DQST está no tratamento dos termos fonte. No DQMoM, a função distribuição é discretizada em $N$ pesos e $N$ abscissas, e as equações para a evolução temporal dos mesmos é obtida a partir da conservação dos $2 N$ momentos da equação de balanço populacional. Desta forma, os termos fontes para os pesos e abscissas são obtidos através da solução de um sistema linear. $O$ problema desta abordagem é que o sistema linear obtido é mal condicionado quando $N$ é grande. Já o método DQST tem como objetivo 
a conservação apenas dos três momentos de ordem mais baixa $\left(\mu_{0}, \mu_{1}\right.$ e $\left.\mu_{2}\right)$, utilizando uma metodologia de conservação e redistribuição local dos momentos, de forma semelhante ao método do pivô móvel, de Kumar e Ramkrishna (2006).

Recentemente, a versão monovariada deste método foi implementada na versão de desenvolvimento do software Star-CCM $+\circledR$ da CD-Adapco ${ }^{\circledR}$, sendo aplicado a problemas de dinâmica dos fluidos computacional (Computational Fluid Dynamics, CFD) (VIKHANSKY e SPLAWSKY, 2015). No entanto, a acurácia do método ainda não foi avaliada de forma apropriada, através da comparação com soluções analíticas. Além disso, não é apresentada nenhuma metodologia de inicialização para o método.

Este trabalho tem como objetivo a avaliação da convergência do método DQST para o caso monovariado, com a avaliação das metodologias de inicialização existentes e o desenvolvimento de uma nova metodologia de inicialização.

\section{METODOLOGIA NUMÉRICA}

\subsection{A Equação de Balanço Populacional}

A equação de balanço populacional é obtida através da aplicação de princípios de conservação à função densidade numérica de partículas, $f(\boldsymbol{x}, \boldsymbol{y}, t)$, que é função do vetor contendo as propriedades internas da partícula $(\boldsymbol{x})$, de propriedades externas que variam espacialmente $(\boldsymbol{y})$, e do tempo (t). Desprezando a dependência funcional das variáveis externas e considerando a distribuição como sendo monovariada e considerando apenas a quebra e agregação de partículas, podemos escrever a PBE como:

$\frac{\partial f(x, t)}{\partial t}=H^{a}(x, t)+H^{b}(x, t)$ onde e $H^{a}$ e $H^{b}$ são os termos fontes de agregação e quebra, respectivamente, definidos por:

$$
\begin{aligned}
& H^{a}(x, t)=\int_{0}^{x_{\max }} a\left(x, x^{\prime}\right) f(x, t) f\left(x^{\prime}, t\right) d x^{\prime} \\
& -\frac{1}{2} \int_{0}^{x} a\left(x, x^{\prime}\right) f\left(x-x^{\prime}, t\right) f\left(x^{\prime}, t\right) d x^{\prime} \\
& H^{b}(x, t)=b(x) f(x, t) \\
& -\int_{x}^{x_{\max }} \vartheta\left(x^{\prime}\right) b\left(x^{\prime}\right) P\left(x \mid x^{\prime}\right) f\left(x^{\prime}, t\right) d x^{\prime}
\end{aligned}
$$

onde $a\left(x, x^{\prime}\right)$ é a frequência de agregação, $b(x)$ é a frequência de quebra, $\vartheta\left(x^{\prime}\right)$ é o número de partículas filhas originadas na quebra da partícula mãe de tamanho $x^{\prime}$ e $P\left(x \mid x^{\prime}\right)$ é a distribuição de probabilidade condicional das partículas filhas originadas na quebra de uma partícula de tamanho $x^{\prime}$.

\subsection{O Método DQST}

Uma definição importante na dedução do método DQST é a definição do operador momento de ordem $k$, dado por:

$$
L_{k}(.)=\int_{0}^{\infty}(.) x^{k} d x
$$

Além disso, o método utiliza uma discretização da função densidade numérica de partículas na forma:

$$
f(x, t) \approx \sum_{i=1}^{N} w_{i}(t) \delta\left(x-\xi_{i}(t)\right)
$$

onde $w_{i}$ e $\xi_{i}$ são os pesos e abscissas da quadratura, respectivamente.

Desta forma, substituindo a aproximação acima na equação de balanço populacional e aplicando o operador momento para $k=0,1,2$ obtemos o seguinte conjunto de equações: 


$$
\begin{aligned}
& \sum_{i=1}^{N} \frac{d w_{i}}{d t}=H_{0}^{\{N\}} \\
& \sum_{i=1}^{N} \frac{d \varsigma_{i}}{d t}=H_{1}^{\{N\}} \\
& 2 \sum_{i=1}^{N} \xi_{i} \frac{d \varsigma_{i}}{d t}-\sum_{i=1}^{N} \xi_{i}^{2} \frac{d w_{i}}{d t}=H_{2}^{\{N\}}
\end{aligned}
$$

onde $H_{k}^{\{N\}}$ é a aproximação usando $N$ pontos de quadratura do momento de ordem $k$ dos termos fonte de quebra e agregação e $\zeta_{i} \doteq \xi_{i} w_{i}$ é a chamada abscissa ponderada. Estes termos são divididos em um termo de nascimento e outro de morte, dados por:

$$
H_{k}^{\{N\}}=B_{k}^{\{N\}}+D_{k}^{\{N\}}
$$

Na agregação, estes termos são dados por:

$$
\begin{aligned}
B_{k}^{a,\{N\}} & =\frac{1}{2} \sum_{j=1}^{N} \sum_{l=1}^{N}\left(\xi_{j}+\xi_{l}\right)^{k} a\left(\xi_{j}, \xi_{l}\right) w_{j} w_{l} \\
D_{k}^{a,\{N\}} & =-\frac{1}{2} \sum_{j=1}^{N} \sum_{l=1}^{N}\left(\xi_{j}^{k}+\xi_{l}^{k}\right) a\left(\xi_{j}, \xi_{l}\right) w_{j} w_{l}
\end{aligned}
$$

Já os termos de nascimento e morte por quebra são dados por:

$$
\begin{aligned}
B_{k}^{b,\{N\}} & =\sum_{j=1}^{N} b\left(\xi_{j}\right) \vartheta\left(\xi_{j}\right) \pi_{k}\left(\xi_{j}\right) w_{j} \\
D_{k}^{b,\{N\}} & =-\sum_{j=1}^{N} b\left(\xi_{j}\right) \xi_{j}^{k} w_{j}
\end{aligned}
$$

Com o termo $\pi_{k}\left(\xi_{j}\right)$ sendo definido como:

$$
\pi_{k}\left(\xi_{j}\right)=\int_{0}^{\xi_{j}} v^{k} P\left(v \mid \xi_{j}\right) d v
$$

A fim de fechar o problema, Vikhansky (2013) analisa os processos de para cada partícula representativa $\left\{\xi_{i}, w_{i}\right\}$ de forma individual. A discretização temporal método de Euler explícito fornece as seguintes equações:

$$
\begin{aligned}
w_{i}^{(t+\Delta t)}= & w_{i}^{(t)}+\left[B_{0}^{a,(i)}(t)-D_{0}^{a,(i)}(t)+\right. \\
& \left.+B_{0}^{b,(i)}(t)-D_{0}^{b,(i)}(t)\right] \Delta t \\
\varsigma_{i}^{(t+\Delta t)}= & s_{i}^{(t)}+\left[B_{1}^{a,(i)}(t)-D_{1}^{a,(i)}(t)+\right. \\
+ & \left.B_{1}^{b,(i)}(t)-D_{1}^{b,(i)}(t)\right] \Delta t
\end{aligned}
$$

onde $D_{k}^{a,(i)}$ e $D_{k}^{b,(i)}$ são os termos fonte de momento de ordem $k$ devido, respectivamente, a agregação e a quebra da partícula $i$, enquanto que $B_{k}^{a,(i)}$ e $B_{k}^{b,(i)}$ são termos fonte de momento de ordem $k$ por nascimento de partículas por, respectivamente, agregação e quebra, que são atribuídos a partícula $i$. Analisando o processo de morte por agregação da partícula $i$, temos que:

$$
D_{k}^{a,(i)}=-\xi_{i}^{k} \sum_{j=1}^{N} a\left(\xi_{i}, \xi_{j}\right) w_{i} w_{j}
$$

Analogamente, os termos fonte de momentos oriundos da morte por quebra da partícula $i$ são:

$$
D_{k}^{b,(i)}=-b\left(\xi_{i}\right) \xi_{i}^{k} w_{i}
$$

No entanto, os termos fonte de momentos devidos ao nascimento de partículas necessitam de um tratamento especial, uma vez que as partículas nascidas não correspondem a nenhuma partícula já existente na distribuição discretizada. Assim, cada termo de nascimento é tratado como uma taxa de formação de $w^{*}$ partículas de tamanho $\xi^{*}$. A determinação dos valores de $w^{*} \mathrm{e} \xi^{*}$ depende do tipo de processo, podendo inclusive ser utilizadas várias partículas representativas para um dado evento. Em seguida, cada partícula filha gerada é redistribuída entre um par de partículas representativas da distribuição $\left[\left\{\xi_{i}, w_{i}\right\},\left\{\xi_{i+1}, w_{i+1}\right\}\right]$, tal que $\xi_{i}<\xi^{*}<\xi_{i+1}$. Neste processo, a fim de garantir a conservação do segundo momento, se a partícula formada for 
maior que a última abscissa, ou seja, $\xi_{N}<\xi^{*}$, a partícula é atribuída ao par $\left[\left\{\xi_{N-1}, w_{N-1}\right\},\left\{\xi_{N}, w_{N}\right\}\right]$, e caso a partícula formada seja menor que a primeira abscissa, ou seja, $\xi^{*}<\xi_{1}$, a partícula é atribuída ao par $\left[\left\{\xi_{1}, w_{1}\right\},\left\{\xi_{2}, w_{2}\right\}\right]$. Assim, para o nascimento por agregação, a redistribuição é escrita como:

$$
\begin{aligned}
B_{0}^{a,(i)}= & K_{i}\left(\xi^{*}\right) w^{*} \\
B_{0}^{a,(i+1)} & =\left[1-K_{i}\left(\xi^{*}\right)\right] w^{*} \\
B_{1}^{a,(i)}= & \frac{K_{i}\left(\xi^{*}\right)}{2}\left(\xi_{i}+\xi_{i+1}\right) w^{*}+ \\
& +\frac{r_{i}^{2}}{2}\left(\xi_{i}-\xi_{i+1}\right) K_{i}\left(\xi^{*}\right) w^{*} \\
B_{1}^{a,(i+1)} & =w^{*} \xi^{*}-B_{1}^{a,(i)}
\end{aligned}
$$

onde, na formulação monovariada de Vikhansky e Splawski (2015) a função $K_{i}\left(\xi^{*}\right)$ correspondente ao intervalo $i$ tem a forma:

$$
K_{i}\left(\xi^{*}\right)=\left\{\begin{array}{cc}
\left(2-r_{i}\right) r_{i}, \quad r_{i}<0 \\
r_{i}, & 0 \leq r_{i} \leq 1 \\
r_{i}^{2}, & 1 \leq r_{i}
\end{array}\right.
$$

onde,

$$
r_{i}=\frac{\xi_{i+1}-\xi^{*}}{\xi_{i+1}-\xi_{i}}
$$

É possível mostrar que utilizando as equações acima o segundo momento é conservado durante a redistribuição. Os termos de nascimento por agregação são descritos pela formação de $N^{2}$ partículas filhas, obtido pela combinação das partículas mãe duas a duas. Com isso, as partículas representativas geradas na agregação das partículas $j$ e $l$ são dadas por:

$$
\begin{aligned}
& w_{j l}^{*}=\left(1-\frac{1}{2} \delta_{j l}\right) a\left(\xi_{j}, \xi_{l}\right) w_{j} w_{l} \\
& \xi_{j l}^{*}=\xi_{j}+\xi_{l}
\end{aligned}
$$

O termo de nascimento por quebra também recebe um tratamento especial. Neste caso, partimos da análise da distribuição de partículas filhas geradas na quebra da partícula mãe de índice $j$, que é dado por:

$$
B^{b,\{j\}}(x)=\vartheta\left(\xi_{j}\right) b\left(\xi_{j}\right) P\left(x \mid \xi_{j}\right) w_{j}
$$

Vikhansky e Splawski (2015) realizam a discretização da distribuição de partículas filhas em $M^{\prime}$ partículas representativas:

$$
P\left(x \mid \xi_{j}\right) \approx \sum_{l=1}^{M^{\prime}} w_{l}^{*,\{j\}} \delta\left(x-\xi_{l}^{*,\{j\}}\right)
$$

levando ao nascimento de $M^{\prime}$ partículas representativas com fonte de momentos de ordem $k$ dada por $\left(\xi_{l}^{*,\{j\}}\right)^{k} w_{l}^{*,\{j\}} \vartheta\left(\xi_{j}\right) b\left(\xi_{j}\right) w_{j}$. Entretanto, a metodologia usada na discretização e o número de partículas $M^{\prime}$ não foram especificados nos testes realizados por Vikhansky e Splawski (2015), de forma que neste trabalho foram implementadas e testadas diferentes estratégias. Uma delas parte da discretização da distribuição de partículas filhas em duas partículas representativas $\xi_{1}^{*,\{j\}} \mathrm{e}$ $\xi_{2}^{*,\{j\}}$, de mesmo peso $w^{*,\{j\}}$, que são calculadas a partir dos 3 momentos de ordem mais baixa:

$$
\begin{aligned}
\mu_{0}^{b,\{j\}}= & \int_{0}^{\xi_{j}} P\left(x \mid \xi_{j}\right) d x=2 w^{*,\{j\}} \\
\mu_{1}^{b,\{j\}}= & \int_{0}^{\xi_{j}} P\left(x \mid \xi_{j}\right) x d x= \\
& =\left(\xi_{1}^{*,\{j\}}+\xi_{2}^{*,\{j\}}\right) w^{*,\{j\}} \\
\mu_{2}^{b,\{j\}}= & \int_{0}^{\xi_{j}} P\left(x \mid \xi_{j}\right) x^{2} d x= \\
= & {\left[\left(\xi_{1}^{*,\{j\}}\right)^{2}+\left(\xi_{2}^{*,\{j\}}\right)^{2}\right] w^{*,\{j\}} }
\end{aligned}
$$

É possível resolver o sistema acima para obter:

$$
w^{*,\{j\}}=\frac{\mu_{0}^{b,\{j\}}}{2}
$$


$\xi_{1}^{*,\{j\}}=$

$=\frac{\mu_{1}^{b,\{j\}}+\sqrt{4 \mu_{0}^{b,\{j\}} \mu_{2}^{b,\{j\}}-\left(\mu_{1}^{b,\{j\}}\right)^{2}}}{2 \mu_{0}^{b,\{j\}}}$

$\xi_{2}^{*,\{j\}}=\frac{2 \mu_{1}^{b,\{j\}}}{\mu_{0}^{b,\{j\}}}-\xi_{1}^{*,\{j\}}$

Uma opção na discretização da distribuição de partículas filhas é a aplicação do algoritmo de Chebschev modificado (GAUTSCHI, 2004), que retorna um conjunto de $M^{\prime}$ pesos e abscissas que reproduz exatamente os momentos de ordem $k=$ $0, \ldots, 2 M^{\prime}-1$ da distribuição, na forma:

$$
\begin{aligned}
\mu_{k}^{b,\{j\}}=\sum_{l=1}^{M \prime} w_{l}^{*,\{j\}} & \left(\xi_{l}^{*,\{j\}}\right)^{k}, \\
& \text { para } k \\
& =0, \ldots, 2 M^{\prime}-1
\end{aligned}
$$

\subsection{Metodologia de suavização}

A metodologia de suavização é a responsável pela adaptatividade do método, e tem como objetivo a distribuição igualitária do primeiro momento entre os pivôs durante toda a solução transiente da equação de balanço populacional. Este procedimento permite que os pesos e abscissas da distribuição se adaptem às modificações, diminuindo o gasto de recursos computacionais usados na evolução de partículas pouco importantes, aumentando a eficiência computacional do método. Assim, considerando que cada abscissa possui dois vizinhos de índice $i-1$ e $i+1$, escrevemos as seguintes equações:

$$
\begin{aligned}
\Delta w_{i} & =\kappa_{i}-\kappa_{i-1} \\
\Delta S_{i}= & \frac{1}{2}\left(\xi_{i+1}+\xi_{i}\right) \kappa_{i} \\
& \quad-\frac{1}{2}\left(\xi_{i-1}+\xi_{i}\right) \kappa_{i-1}
\end{aligned}
$$

onde $\kappa_{i}$ é o momento de ordem 0 transferido da partícula $i+1$ para a partícula $i$. Para tornar o equacionamento geral definimos $\kappa_{0}$ e $\kappa_{N}$ como sendo nulos, uma vez que o primeiro pivô não tem vizinhos do lado esquerdo e o ultimo não possui vizinhos do lado direito. Os parâmetros $\kappa_{i}$ são calculados com base na minimização do somatório dos quadrados das abscissas ponderadas após aplicação dos incrementos, escrita como:

$$
\begin{array}{r}
\boldsymbol{\kappa}=\arg \min _{\kappa}\left[\frac { 1 } { 2 } \sum _ { \mathrm { i } = 1 } ^ { \mathrm { N } - 1 } \left(s_{\mathrm{i}}+\frac{1}{2}\left(\xi_{i+1}+\xi_{i}\right) \kappa_{i}\right.\right. \\
\left.\left.-\frac{1}{2}\left(\xi_{i-1}+\xi_{i}\right) \kappa_{i-1}\right)^{2}\right]
\end{array}
$$

Aplicando a condição de gradiente nulo para minimização da equação acima, obtemos um sistema linear com matriz tridiagonal dado por:

$$
a_{i} \kappa_{i-1}+b_{i} \kappa_{i}+c_{i} \kappa_{i+1}=d_{i}
$$

onde,

$$
\begin{aligned}
& a_{i}=-\left(\xi_{i-1}+\xi_{i}\right)\left(\xi_{i+1}+\xi_{i}\right) \\
& b_{i}=2\left(\xi_{i+1}+\xi_{i}\right)^{2} \\
& c_{i}=-\left(\xi_{i+1}+\xi_{i}\right)\left(\xi_{i+2}+\xi_{i+1}\right) \\
& d_{i}=2\left(\xi_{i+1}+\xi_{i}\right)\left(\zeta_{i+1}-\zeta_{i}\right) \\
& a_{0}=c_{N-1}=0
\end{aligned}
$$

Após a solução do sistema linear, os incrementos são aplicados de acordo com as seguintes equações:

$$
\begin{aligned}
w_{i}^{(n)}= & w_{i}^{(o)}+\kappa_{i}-\kappa_{i-1} \\
\varsigma_{i}^{(n)}=\varsigma_{i}^{(o)} & +\frac{1}{2}\left(\xi_{i+1}+\xi_{i}\right) \kappa_{i} \\
& -\frac{1}{2}\left(\xi_{i-1}+\xi_{i}\right) \kappa_{i-1}
\end{aligned}
$$

Quando uma formulação peso-abscissa é usada, a equação de evolução da abscissa se torna a seguinte:

$$
\begin{aligned}
\xi_{i}^{(n)} & =\frac{\xi_{i}^{(o)} w_{i}^{(o)}}{w_{i}^{(n)}}+ \\
+ & \frac{1}{2} \frac{\left(\xi_{i+1}+\xi_{i}\right) \kappa_{i}-\left(\xi_{i-1}+\xi_{i}\right) \kappa_{i-1}}{w_{i}^{(n)}}
\end{aligned}
$$




\subsection{Metodologia de Inicialização}

Para comparação da solução obtida pelo método DQST com soluções analíticas em que a condição inicial é fornecida como uma distribuição contínua, a aproximação discreta da mesma deve ser calculada. Existem diversas metodologias de discretização para distribuições mono e multivariadas. Uma possibilidade seria a aplicação do método de Chebschev modificado, que dados $2 \mathrm{~N}$ momentos da distribuição inicial, a metodologia retorna os $N$ pesos e abscissas que retornam os $2 \mathrm{~N}$ momentos de forma exata. Entretanto, este algoritmo falha para $N$ grande $(\approx 15)$, de forma que este método não seria útil na analise da convergência do método DQST para $N$ grande. Assim, foi desenvolvida uma metodologia de discretizacao baseada no método das classes, mas que conserva somente os momentos de ordem 0 e 1 da distribuição, a fim de se testar a convergência do método DQST para $N$ grande. Para obtermos uma metodologia de inicialização compatível com a de suavização, buscamos uma aproximação que distribui igualmente o primeiro momento entre os pesos e abscissas, de forma que:

$$
\xi_{i}=\frac{\Delta \mu_{1}}{w_{i}}, \quad \forall i
$$

onde $\Delta \mu_{1}$ é o momento de primeira ordem atribuído a cada par peso-abscissa, calculado por:

$$
\Delta \mu_{1}=\frac{\mu_{1}}{N}
$$

O cálculo dos pesos e abscissas é baseado na definição dos momentos seccionais de Kumar e Ramkrishna (1996). Para a definição da primeira abscissa fazemos:

$$
\Delta \mu_{1}=\int_{0}^{x_{1}} x f(x) d x
$$

O lado direito da equação acima deve ser resolvido para obtenção do valor de $x_{1}, \mathrm{o}$ que pode ser feito de forma numérica ou analítica. Uma vez que $x_{1}$ é obtido, calculamos o peso relativo ao primeiro intervalo por:

$$
w_{1}=\int_{0}^{x_{1}} f(x) d x
$$

A metodologia é aplicada sequencialmente para cada intervalo, e o método pode ser generalizado por:

$$
\begin{aligned}
& \Delta \mu_{1}=\int_{x_{i-1}}^{x_{i}} x f(x) d x ; i=1, \ldots, N-1 \\
& w_{i}=\int_{x_{i-1}}^{x_{i}} f(x) d x \\
& \xi_{i}=\frac{\Delta \mu_{1}}{w_{i}}
\end{aligned}
$$

A fim de se melhorar a robustez do método, no último par peso-abscissa faz-se:

$$
\begin{aligned}
& w_{N}=\mu_{0}-\sum_{i=1}^{N-1} w_{i} \\
& \xi_{N}=\frac{\Delta \mu_{1}}{w_{N}}
\end{aligned}
$$

\subsection{Procedimento Numérico}

As integrais que surgem no procedimento de inicialização podem ser calculadas de forma analítica ou numérica. Nos testes realizados, estas integrais foram resolvidas numericamente através da rotina gsl_integration_qng da biblioteca GSL (Gnu Scientific Lìbrary) (GALASSI et al, 2015). As equações não-lineares associadas ao cálculo dos intervalos foram resolvidas através do método da secante. Além disso, com a finalidade de avaliar os erros oriundos do procedimento de suavização, uma forma iterativa com sub-relaxação do mesmo foi 


\section{ONEMP}

implementada. O procedimento iterativo é aplicado da seguinte forma:

1. Definir número de repetições, $N_{\text {loop }}$

2. Fazer $N_{\text {loop }}$ vezes:

2.1 Calcular $\kappa_{i}$ através da solução do sistema linear

2.2 Fazer $\kappa_{i}^{*}=\frac{\kappa_{i}}{N_{\text {loop }}}$

2.3 Aplicar a suavização com $\kappa_{i}^{*}$

Finalmente, a solução transiente da EBP é obtida pela aplicação das etapas descritas nas seções anteriores de acordo com o seguinte algoritmo:

1. Definição dos modelos de quebra e agregação, condição inicial, número de pontos de quadratura, metodologia de discretizacao da quebra, passo de tempo $(\Delta t)$ e tempo total de simulação $\left(t^{\text {final }}\right)$.

2. Inicialização utilizando a metodologia mostrada na Seção 2.4 ou o algoritmo de Chebschev modificado.

3. Aplicação da metodologia de suavização à condição inicial, uma ou $N_{\text {loop }}$ vezes.

4. Repetir para cada passo de tempo:

4.1 Cálculo dos termos fonte de quebra e agregação.

4.2 Incremento dos pesos e abscissas.

4.3 Solução do sistema linear para obtenção dos $\kappa_{i}$ e aplicação da metodologia de suavização.

4.4 Escrita da solução em arquivo e dos erros em relação a solução analítica, se houver.

4.5 se $t<t^{\text {final }}$, fazer $t=t+\Delta t \quad \mathrm{e}$ volta para 3.1.

se $t \geq t^{\text {final }}$, terminar o algoritmo.

\section{RESULTADOS E DISCUSSÃO}

\subsection{Metodologia de Inicialização}

Nesta seção o comportamento da metodologia de inicialização desenvolvida será comparado ao método de Chebschev modificado. Na primeira iteração do algoritmo, após a aplicação da metodologia de inicialização, é realizada pela primeira vez a metodologia de suavização. Desta forma, os erros reportados nesta seção para cada metodologia de inicialização são os erros obtidos após aplicação da suavização. A influência do número de repetições no erro obtido na inicialização da distribuição $f(x, 0)=$ $e^{-x}$ pela aplicação do algoritmo de Chebschev modificado é mostrada na Fig. 1. É possível observar que a utilização de repetições na metodologia de suavização diminui o erro obtido. Porém, mesmo utilizando um número alto de repetições, o erro relativo ao segundo momento não zera, sendo o erro mínimo obtido da ordem de $10^{-2}$ para 4 pontos de quadratura e de $10^{-1}$ para 16 pontos, de forma que se observa um aumento do erro com o número de pontos de quadratura.

Figura 1 - Comportamento do algoritmo de Chebschev modificado após a suavização.

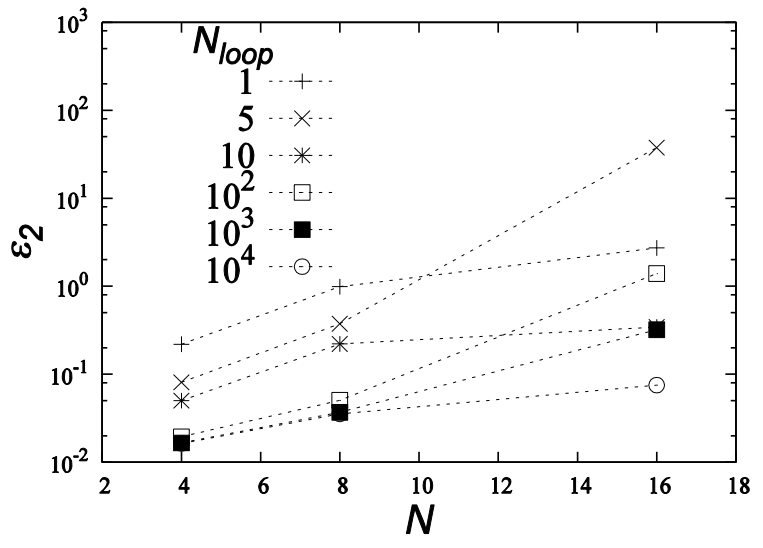

Os erros mínimos obtidos pela aplicação do algoritmo de Chebschev foram comparados com a metodologia apresentada na Seção 2.4. Os erros obtidos são mostrados na Tabela 1. Para $N=2$, o algoritmo de Chebschev apresenta erro nulo para o segundo e o terceiro momento, uma vez que a solução obtida, além de conservar os quatro primeiros momentos da distribuição, possuía uma distribuição igual do primeiro momento entre as abscissas, de forma que não há necessidade 
de aplicação da suavização. Por outro lado, o erro obtido na aplicação da metodologia desenvolvida diminui com o aumento de $N$, em oposição ao comportamento do algoritmo de Chebschev modificado. Com $N=16$, o erro obtido pela nova metodologia é menor tanto para o momento de segunda ordem quanto para o de terceira ordem. Outra vantagem da nova metodologia é a aplicabilidade da mesma para $N$ ainda maiores. A Fig. 2 mostra o comportamento do erro dos momentos de segunda e terceira ordem para a nova metodologia com $N$ variando entre 2 e 1024 .

Tabela 1 - Comparação entre o método de Chebschev modificado (M1) e a metodologia desenvolvida (M2) após a suavização.

\begin{tabular}{ccccc} 
& $\varepsilon_{2}(\%)$ & \multicolumn{3}{c}{$\varepsilon_{3}(\%)$} \\
$N$ & M1 & M2 & M1 & M2 \\
\hline 2 & 0 & 17,7 & 0 & 37,1 \\
4 & 1,63 & 6,55 & 2,11 & 17,3 \\
8 & 3,54 & 2,50 & 3,17 & 8,24 \\
16 & 7,51 & 0,98 & 14,1 & 3,98
\end{tabular}

Figura 2 - Comportamento dos erros dos momentos de segunda e terceira ordem utilizando a nova metodologia de inicialização (M2).

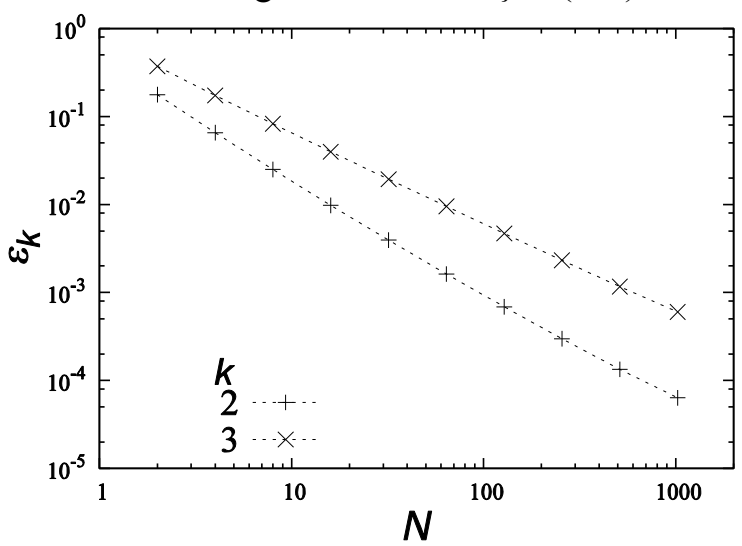

\subsection{Agregação pura}

Neste caso os testes foram realizados para frequência de agregação dada por $a\left(x, x^{\prime}\right)=1$. A metodologia de inicialização utilizada é a M2, sem sub-relaxação. Os resultados para convergência com o número de pontos de quadratura utilizando um passo de tempo $\Delta t=10^{-4}$ são mostrados na Figura 3. Os erros obtidos para o momento de ordem 0 são inicialmente da ordem de $10^{-12}$, estabilizando em $10^{-5}$ durante a integração temporal, e não variam com o número de pontos de quadratura. Realizando uma convergência com o passo de tempo foi possível observar que este erro é relativo à integração temporal. $\mathrm{O}$ momento de primeira ordem é totalmente conservado, com erros na faixa entre $10^{-14}$ e $10^{-16}$.

Figura 3 - Comportamento dos erros dos momentos de ordem 0,1 e 2 para o caso de agregação pura com frequência de agregação constante.

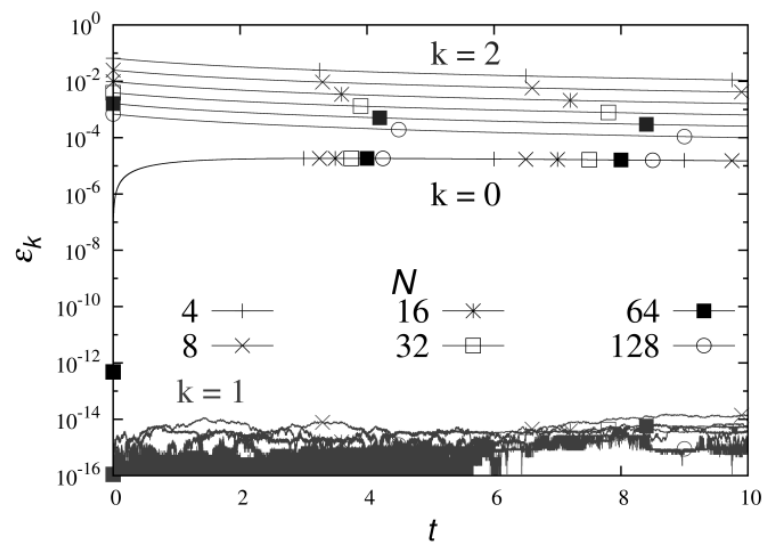

Já os erros dos momentos de segunda ordem possuem uma grande influência com o número de pontos de quadratura utilizados, variando entre $10^{-1}$ para 4 pontos de quadratura até $10^{-4}$ com 128 pontos de quadratura. Foi possível observar que os erros permaneceram praticamente constantes durante a integração temporal, de forma que os erros obtidos são oriundos exclusivamente da metodologia de inicialização empregada. Os perfis dos momentos $\mu_{0} \quad$ e $\quad \mu_{1} \quad$ apresentaram comportamentos semelhantes aos mostrados na Figura 3 para os outros casos teste avaliados, não sendo influenciados pelo número de pontos de quadratura utilizado. 
Assim, as análises apresentadas daqui em diante considerarão apenas o momento de segunda ordem.

\subsection{Quebra e Agregação Simultâneas}

Nesta seção será avaliado o caso com agregação e quebra simultâneas, para o caso particular cuja solução analítica foi obtida por McCoy e Madras (2002), com condição inicial $f(x, 0)=e^{-x}, \quad b(x)=x, \quad P\left(x \mid x^{\prime}\right)=H\left(x^{\prime}-x\right) / x^{\prime}$, $\vartheta\left(x^{\prime}\right)=2$ e $a\left(x, x^{\prime}\right)=1$, o que corresponde ao caso de equilíbrio entre quebra e agregação, em que a solução é invariante. A metodologia de inicialização utilizada é a M1, sem subrelaxação. O passo de tempo utilizado foi $\Delta t=$ $10^{-4}$.

Foi realizada a análise da convergência com o número de pontos de quadratura na distribuição $f(x, 0)$ e na discretização da distribuição condicional de partículas filhas, $P\left(x \mid x^{\prime}\right)$. A Figura 4 mostra a influência do número de pontos de quadratura para o caso analisado utilizando a metodologia de discretização para $P\left(x \mid x^{\prime}\right)$ que utiliza duas partículas de mesmo peso. É possível observar erros entre $10^{-2}$ e $10^{-3}$ em todos os casos. Este resultado mostra a necessidade de avaliação da influência da discretização da distribuição de partículas filhas. Para isso, foi realizada a discretização usando o método de Chebschev modificado com $M^{\prime}=1, \ldots, 4$ pontos de quadratura. A Figura 5 mostra os erros obtidos para $N=8$. É possível observar a diminuição do erro com o aumento de $M^{\prime}$, de forma que os erros estão na faixa entre $10^{-1}$ e $10^{-3}$. A Figura 6 mostra a mesma análise para $N=128$. Os erros obtidos são menores que no caso anterior, sendo da ordem de $10^{-4}$ para $M^{\prime}=4$, mostrando que o número de pontos de quadratura usado em cada discretização tem grande influência no erro do segundo momento.
Figura 4 - Erro dos momentos de segunda ordem com o número de pontos de quadratura para solução invariante de McCoy e Madras (2003).

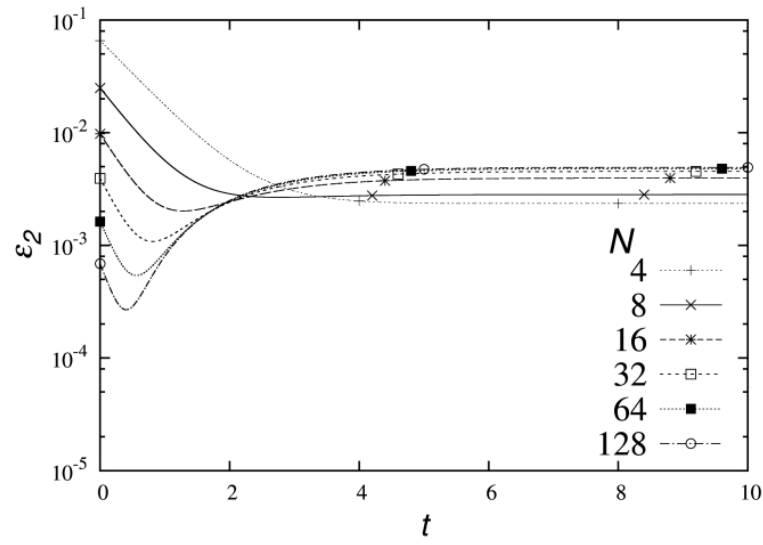

Figura 5 - Erros dos momentos de segunda ordem com o número de pontos de quadratura usados na discretização da distribuição de partículas filhas $\operatorname{com} N=8$.

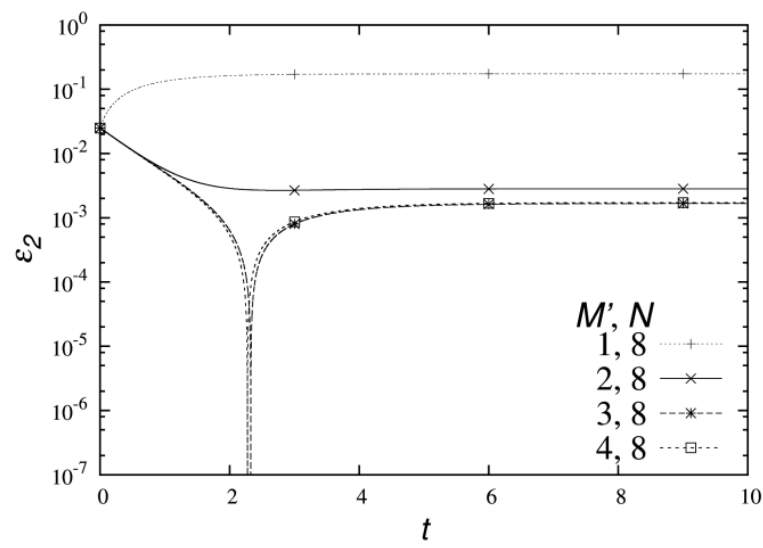

Figura 6 - Erros dos momentos de segunda ordem com o número de pontos de quadratura usados na discretização da distribuição de partículas filhas com $N=128$

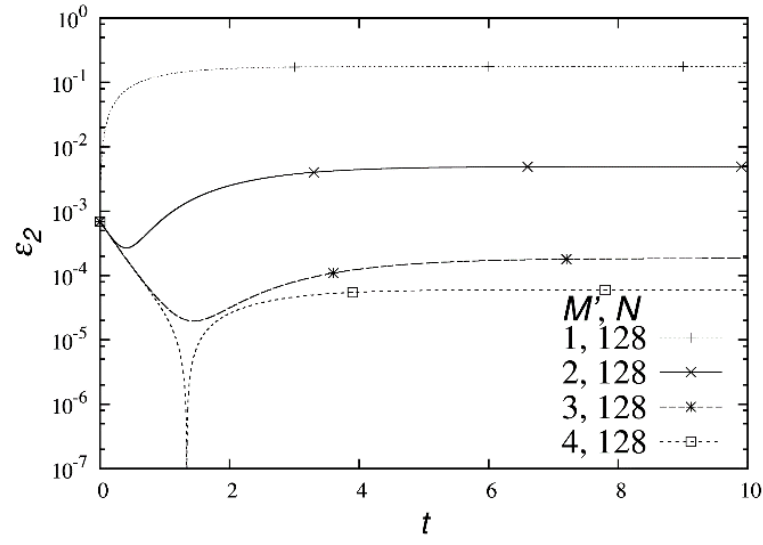




\section{CONCLUSÃO}

O método DQST foi implementado e os resultados obtidos foram comparados com soluções analíticas reportadas na literatura. $\mathrm{O}$ erro do momento de segunda ordem para o caso de agregação pura foi relativamente alto, mas ocorre principalmente devido à metodologia de inicialização, já que não foi possível observar acúmulo de erros durante a solução transiente da EBP. No caso com quebra e agregação simultâneas, foi possível observar uma grande influência da discretização da distribuição condicional de partículas filhas na acurácia do método. Trabalhos futuros utilizarão estes resultados preliminares na proposição de modificações ao método, com foco na melhora da acurácia do mesmo no cálculo do momento de segunda ordem.

\section{NOMENCLATURA}

$a$ frequência de agregação, $T^{-1}$

$b$ frequência de quebra, $T^{-1}$

$f$ função densidade numérica de partículas

$H \quad$ termo fonte da EBP, $T^{-1}$

$M^{\prime} \quad$ número de pontos de quadratura

usados na discretização da distribuição

condicional de partículas filhas geradas na quebra

$P \quad$ distribuição condicional de partículas

filhas geradas na quebra

$w$ peso da discretização da função

densidade numérica

$x \quad$ variável genérica da EBP

$\kappa \quad$ vetor de transporte de número de

partículas na suavização

$\mu \quad$ momento da distribuição

$\xi \quad$ abscissa da discretização da função

densidade numérica

$\varsigma \quad$ abscissa ponderada da discretização da função densidade numérica

$\vartheta \quad$ número de partículas filhas geradas na quebra

\section{REFERÊNCIAS}

MARCHISIO, D. L., FOX, R. O., Computational Models for Polydisperse Particulate and Multiphase Systems. Nova York, Cambridge University Press., 2013.

RAMKRISHNA, D., Population Balances Theory and Applications to Particulate Systems in Engineering. San Diego, Academic Press, 2000.

VIKHANSKY, A., Direct quadrature spanning tree method for solution of the population balance equations, Journal of Aerosol Science, v. 55, p. 78-88. 2013.

VIKHANSKY, A., SPLAWSKI, A., Adaptative multiply size group method for CFD-population balance modelling of polydisperse flows, The Canadian Journal of Chemical Engineering, p. 78-88, 2015.

KUMAR, S., RAMKRISHNA, D., On the solution of population balance equations by discretization-II. A moving pivot technique, Chemical Engineering Science, v. 51, n. 8, p. 1333-1342, 1996.

GAUTSCHI, W. Orthogonal polynomials: computation and approximation, Oxford, Oxford University Press, 2004.

GALASSI, M., DAVIES, J., THEILER, J., GOUGH, B., PRIEDHORSKI, R., JUNGMAN,G., BOOTH, M., GNU Scientific Library Reference Manual (3rd Ed.), url: http://www.gnu.org/software/gsl/, acessado 15/06/2015, às 11:00.

MCCOY, B. J., MADRAS, G., Analytical solution for a population balance equation with aggregation and fragmentation, Chemical Engineering Science, v. 58, pp. 3049-3051, 2003. 